\title{
Ciencia-Tecnología-Sociedad vs. STEM: ¿evolución, revolución o disyunción?
}

\author{
F. Javier Perales Palacios y David Aguilera \\ Departamento de Didáctica de las Ciencias Experimentales. Facultad de Ciencias de la \\ Educación. Universidad de Granada. España
}

[Recibido el 3 de diciembre de 2019, aceptado el 21 de enero de 2020]

El presente trabajo trata de dilucidar las posibles relaciones entre dos movimientos educativos de indudable relevancia en la Didáctica de las Ciencias Experimentales a lo largo de las últimas décadas, uno de ellos más pionero, conocido como Ciencia-Tecnología-Sociedad (CTS) y otro más reciente, Ciencia-Tecnología-Ingeniería-Matemáticas (STEM). Para ello partimos de una concreción de sus orígenes, objetivos, características, líneas de trabajo y propuestas didácticas en cada uno de ellos. A continuación, nuevamente apoyados en una revisión bibliográfica, establecemos las comparaciones entre ambos a partir de los apartados referidos anteriormente. Tal comparación nos lleva a poner en cuestión la vinculación entre ambos y la originalidad de la propuesta STEM, junto con las dificultades que su implementación conlleva.

Palabras clave: Ciencia-Tecnología-Sociedad; educación STEM; didáctica de las ciencias experimentales; formación del profesorado; currículo.

\section{Science-Technology-Society vs. STEM: Evolution, revolution or disjunction?}

The present work tries to elucidate the possible relations between two educational movements of unquestionable relevance in Science Education throughout the last decades, one of them more pioneer, known as Science-Technology-Society (STS) and another recent, Science-TechnologyEngineering-Mathematics (STEM). For this we start from a concretion of its origins, objectives, characteristics, lines of work and didactic proposals in each of them. Then, again supported by a bibliographic review, we establish the comparisons between the two from the sections referred to above. Such comparison leads us to question the relationship between the two and the originality of the STEM proposal, together with the difficulties that its implementation entails.

Keywords: Science-Technology-Society; STEM education; science education; teacher training; curriculum.

Para citar el artículo. Perales-Palacios, F.J. y Aguilera, D. (2020). Ciencia-Tecnología-Sociedad vs. STEM: ¿evolución, revolución o disyunción? Ápice. Revista de Educación Científica, 4(1), 1-15. DOI: https://doi.org/10.17979/arec.2020.4.1.5826

Contacto.fperales@ugr.es, davidaguilera@ugr.es 


\section{Introducción}

La Didáctica de las Ciencias Experimentales (DCE) es hoy por hoy una disciplina compleja tras décadas de consolidación epistemológica, social y profesional. Ello conlleva la proliferación de líneas de investigación que ponen el énfasis en algunas de sus múltiples variantes. Dichas líneas intentan aislar determinadas variables de manera que pueda visualizarse el efecto de las mismas en los resultados deseados, normalmente la mejora de la enseñanza y aprendizaje de las Ciencias. No resulta fácil englobar dichas líneas en categorías más generales, aunque en un esfuerzo de simplificación podríamos hablar de las referentes a: el alumno, el profesor, los materiales y recursos de enseñanza, o el contexto.

Con relación a estas dos últimas categorías, sobresalen dos tendencias surgidas en el mundo occidental que centran su atención, respectivamente, en la naturaleza social de la Ciencia y la Tecnología (CTS) y, más recientemente, aquella que amplía las relaciones interdisciplinares a las Matemáticas y las Ingenierías $\left(\mathrm{STEM}^{1}\right)$ con el fin último de incrementar las vocaciones hacia las mismas en los países occidentales frente al desafío de las nuevas potencias económicas. Para este último caso se ha comenzado a acuñar el acrónimo STEAM para dar cabida a la A de Arte, entendido en un sentido amplio (pintura, música, literatura...).

En ambos casos la incidencia en la comunidad educativa e investigadora ha sido o comienza a ser de la suficiente entidad como para obligar a preguntarnos por los rasgos comunes y diferenciadores de los movimientos CTS y STEM o, yendo más lejos, si se puede considerar que STEM es en cierta medida el resultado de una evolución del movimiento CTS o, por el contrario, representa una revolución respecto a aquel. En el primer caso tendría una connotación más próxima a la filosofía de Stephen Toulmin (1972), quien entiende la comprensión humana como la suma de varias empresas racionales en evolución (Chamizo, 2006) y en el segundo a la de Tomas Khun (1962) y su célebre teoría de las revoluciones científicas.

Por otro lado, y desde un punto de vista filosófico, también CTS y STEM (y especialmente STEAM) pueden considerarse manifestaciones de corrientes nacidas en el siglo pasado que tratan de poner el acento en un pensamiento global ("noosfera") y en la necesidad de analizar nuestro mundo desde la perspectiva de la complejidad. Entre los referentes de esta perspectiva podemos citar, respectivamente, a los filósofos franceses Theilard de Chardin (Montovani, 2020) y Edgar Morin (1990), en el caso de este último sus aportaciones al pensamiento complejo están dando lugar a la aceptación de las llamadas "Ciencias de la Complejidad".

El objetivo de este trabajo consiste en tratar de ofrecer una respuesta fundamentada a la cuestión anterior, a partir de una reflexión sobre las aportaciones de la bibliografía a la definición de ambos movimientos y haciendo uso de una perspectiva comparada.

\section{El movimiento CTS}

Desde el punto de vista epistemológico, CTS hunde sus raíces en los vínculos entre Ciencia y Sociedad (p. ej., Bernal, 1967) y, por otro lado, en el propio desarrollo tecnológico de la humanidad y su incidencia en la forma de vida y costumbres. Visto desde una perspectiva global, CTS trata de integrar y poner de manifiesto las conexiones mutuas entre los tres vértices del triángulo, que en muchas ocasiones pasan desapercibidas o están lastradas

1 Dado su uso extendido en español, mantenemos la nomenclatura STEM en lugar de su equivalente en nuestro idioma, como ocurre con CTS. 
por falsas creencias, tales como que la Tecnología es subsidiaria de la Ciencia o que la Sociedad no puede condicionar la Ciencia.

Aunque existen precedentes en los años 70 del pasado siglo (Gallagher, 1971 y Hurd, 1975, citados en Aikenhead, $2003^{2}$ ), incluso con programas universitarios con dicha orientación, es en la década de los 80 cuando se producen una serie de informes en distintos países occidentales reclamando que la educación científica se amplíe a los estudiantes que no van a cursar carreras científicas, lo que derivó en el lema de "Ciencia para todos" y que, a su vez, propició la aparición de proyectos que enfatizaban "la aplicación tecnológica de la Ciencia en la Sociedad moderna" o CTS (Fensham, 2016, p. 167). En cualquier caso, la T de Tecnología fue la más rezagada en su incorporación real al acrónimo al contar con ciertas reticencias entre la comunidad científica, seguramente lastrada por su formación inicial más científica que tecnológica, lo que ha perjudicado igualmente a la implementación de su vertiente educativa -Educación Tecnológica-.

A este respecto, Siu, Wong y Feng (2010) señalan como elemento clave la publicación en los Estados Unidos en el año 1996 del informe denominado "Technology for All Americans: A Rationale and Structure for the Study of Technology" que proporcionó el fundamento filosófico para los "Standards for Technological Literacy: Content for the Study of Technology".

Posteriormente, el acrónimo CTS se amplió por algunos autores a CTSA, incluyendo la A de dimensión Ambiental (p. ej., en nuestro país Vilches, Gil y Praia, 2011; Vilches y Gil, 2003). Para Yalaki (2016) el objetivo principal de la educación CTSA es empoderar a las personas, ayudándolas a alcanzar logros científicos y poder tomar decisiones informadas sobre temas relacionados con la Ciencia y la Tecnología que influyen en la sociedad.

Como señalara Acevedo (1997), CTS es ante todo una propuesta educativa innovadora que plantea un cambio radical en el currículo escolar, que contempla la formación en valores y que facilita la participación ciudadana en las implicaciones de la Ciencia y la Tecnología.

Si nos preguntamos de un modo más concreto por las características de la educación CTS podríamos avanzar las siguientes: (a) desde un punto de vista curricular se asume una naturaleza interdisciplinar, vinculando Ciencia y Tecnología, y asumiendo unas relaciones estrechas entre ambas tanto a nivel teórico como aplicado, admitiendo diversos grados de integración de CTS en el currículo escolar (Aikenhead, 2003); (b) se parte del supuesto de que tanto la Ciencia y la Tecnología no pueden estudiarse sin considerar el contexto social en el que se desarrollan; (c) en consonancia con lo anterior, se promueve una participación de los ciudadanos (no solo de los estudiantes) en la toma de decisiones que les pueden afectar en estos ámbitos, lo que les aproxima a un concepto de democracia real; (d) los objetivos de aprendizaje incluyen el acercamiento a problemas del mundo real y sus implicaciones sociales, comprendiendo necesariamente conocimientos conceptuales, procedimentales y actitudinales que suelen agruparse en lo que se denomina una alfabetización tecnocientífica, evidentemente necesaria para poder satisfacer el apartado (c); y (e) incrementar los conocimientos científicos y tecnológicos, así como las relaciones y diferencias entre ellos, con el fin de atraer al alumnado hacia las actividades profesionales relacionadas con la Ciencia y la Tecnología (Acevedo, 1997).

La característica (d) implica un desplazamiento hacia el desarrollo por parte de los estudiantes de habilidades transferibles de un mayor nivel cognitivo y que se han denominado como capacidades HCOS (habilidades cognitivas de orden superior de los estudiantes, en español) (Zoller, 2012). En la Figura 1 concretamos dichas capacidades.

2 Este autor detalla en su artículo los precedentes del movimiento CTS a nivel internacional. 


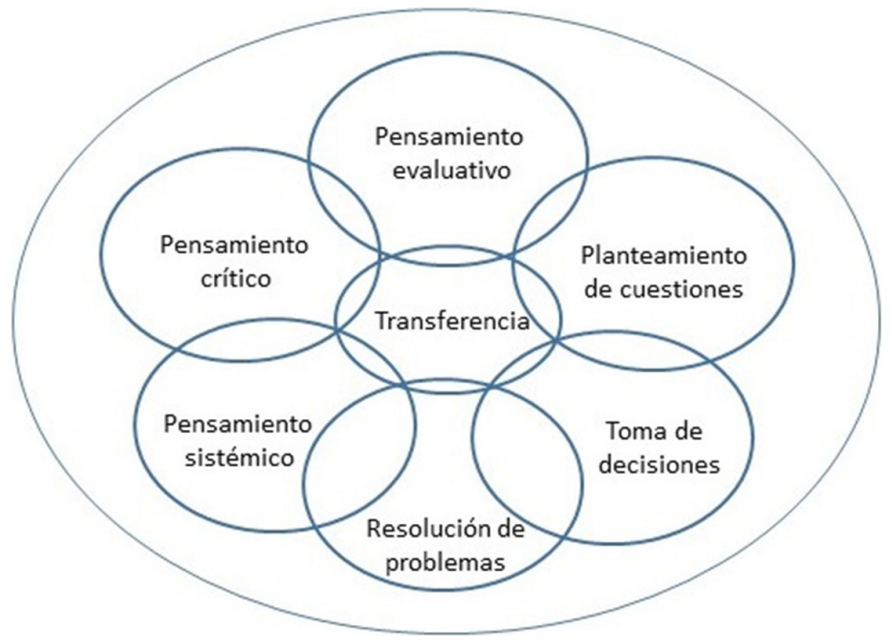

Figura 1. El modelo conceptual HOCS en el contexto de la Educación Científica ${ }^{3}$ (Zoller y Levy Nahum, 2012)

Tras los pioneros trabajos surgidos a nivel internacional (en nuestro país fueron protagonizados por Solbes y Vilches, 1989 y consolidados por Acevedo, 1995, 1996), la investigación ha sido abundante y creciente. Así, por ejemplo, este año se cumple la VII edición del Seminario Iberoamericano CTS y el vigésimo aniversario de su puesta en marcha. A este respecto, Strieder, Bravo y Gil (2017, p. 30) han agrupado las investigaciones en:

a) Trabajos que estudian la evolución de la educación en CTS en términos cuantitativos, líneas temáticas, referencias más utilizadas o autores con mayor producción.

b) Trabajos que abordan las semejanzas y diferencias entre CTS y otras perspectivas como las cuestiones socio-científicas, la enseñanza en contexto o la pedagogía de Paulo Freire.

c) Trabajos que hacen referencia a cómo se ha introducido CTS en los currículos escolares y cuáles han sido sus propósitos y propuestas implícitos.

Una vez esbozados los objetivos educativos derivados de CTS y las diferentes líneas de investigación, cabría preguntarse por las metodologías de enseñanza propuestas para alcanzar dichos objetivos, en conexión con los trabajos agrupados en el apartado c anterior. De la revisión que hemos efectuado podría deducirse que, en general, no puede decirse que se dé una preponderancia de unos métodos sobre otros, aunque podrían agruparse en torno a metodologías activas como denominador común, es decir, aquellas en las que la mayor responsabilidad recae sobre el aprendiz. Dentro de aquellas se mencionan, por ejemplo, el análisis de fuentes de información, aprendizaje basado en proyectos o mediante resolución de problemas abiertos, estudios de caso, actividades extracadémicas (visitas de museos, centros de investigación, participación en actividades comunitarias...) (Kolst $\varnothing, 2001 ;$ Gordillo, 2009). En cuanto a cómo reflejan los libros de texto el enfoque CTS, el resultado parece ser desigual (García-Carmona, 2008; GarcíaCarmona y Criado, 2008). Visto desde otra perspectiva, estas metodologías deberían ser consistentes con las habilidades propuestas por Zoller y Levy Nahum (2012) reflejadas en la Figura 1.

En la Tabla 1 reproducimos una comparación entre la enseñanza tradicional y la basada en CTS.

3 La traducción es nuestra. 
Tabla 1. Diferencia entre orientaciones tradicionales y CTS en Didáctica de las Ciencias (Lawrence, Yager, Sowell, Hancock, Yalaki, y Jablon, 2001, p.17) ${ }^{4}$

\begin{tabular}{|l|l|}
\hline Orientaciones tradicionales & Orientaciones CTS \\
\hline $\begin{array}{l}\text { Los profesores y los libros de texto son las prin- } \\
\text { cipales fuentes de conocimiento }\end{array}$ & $\begin{array}{l}\text { Los estudiantes buscan activamente la in- } \\
\text { formación que usan }\end{array}$ \\
\hline $\begin{array}{l}\text { La Ciencia es abstracta y no tiene relación con } \\
\text { la Tecnología }\end{array}$ & $\begin{array}{l}\text { Los estudiantes ven la Ciencia como una for- } \\
\text { ma de tratar los problemas de la vida diaria }\end{array}$ \\
\hline $\begin{array}{l}\text { Los estudiantes se concentran sobre problemas } \\
\text { que son identificados por el profesor o los libros } \\
\text { de texto }\end{array}$ & $\begin{array}{l}\text { Los estudiantes identifican problemas sobre } \\
\text { ellos mismos o su comunidad y se respon- } \\
\text { sabilizan de resolverlos utilizando la Ciencia }\end{array}$ \\
\hline $\begin{array}{l}\text { Se da una consideración mínima a la capacidad } \\
\text { adaptativa humana }\end{array}$ & $\begin{array}{l}\text { Se enfatizan la adaptación humana y las fu- } \\
\text { turas alternativas }\end{array}$ \\
\hline $\begin{array}{l}\text { No se interpretan los valores en los problemas } \\
\text { de la disciplina }\end{array}$ & $\begin{array}{l}\text { Se consideran las cuestiones relacionadas } \\
\text { con las dimensiones en valores, éticas y mo- } \\
\text { rales de los problemas }\end{array}$ \\
\hline $\begin{array}{l}\text { El currículo está centrado en los libros de texto, } \\
\text { es inflexible; solo se considera el conocimiento } \\
\text { científico válido (y desde una visión limitada del } \\
\text { contenido) }\end{array}$ & $\begin{array}{l}\text { El currículo está centrado en los problemas, } \\
\text { tíficamente }\end{array}$ \\
\hline $\begin{array}{l}\text { La información está enmarcada en el contexto } \\
\text { de la lógica y estructura de la disciplina }\end{array}$ & $\begin{array}{l}\text { La información se enmarca en el contexto } \\
\text { del estudiante como una persona en un en- } \\
\text { torno social/cultural }\end{array}$ \\
\hline
\end{tabular}

Mención aparte merecen algunos enfoques metodológicos y propuestas muy cercanos a los postulados del movimiento CTS, como han sido las Controversias Socio-Científicas (SSI, en inglés) y la Investigación e Innovación Responsables (RRI, en inglés).

Las SSI constituyen problemas sociales reales de naturaleza filosófica y empírica que se utilizan para promover la alfabetización científica funcional y aumentar consiguientemente la comprensión de los estudiantes sobre el conocimiento científico. Chowdhury (2016) afirma que las SSI han demostrado tener éxito al conectar los problemas epistemológicos, morales, emocionales, éticos y culturales con la Ciencia; asimismo han contribuido a comprender la naturaleza de la Ciencia y a centrarse en los aspectos humanísticos y socializadores de las prácticas científicas. Por lo tanto, dicho autor considera que las SSI vienen a complementar algunas carencias del movimiento CTS o CTSA.

Por otro lado, la RRI fomenta la participación de la sociedad en la Ciencia y la innovación, "desde las fases más iniciales" de los procesos de investigación e innovación, para alinear sus resultados con los valores de la sociedad. Se trata de un término amplio que aglutina diferentes aspectos de la relación entre la investigación e innovación y la sociedad: participación ciudadana, acceso abierto, igualdad de género, educación científica, ética y gobernanza (RRI Tools, 2020). A este respecto, Grunwald (2011) destaca que el postulado de la innovación responsable agrega una reflexión ética explícita a los estudios CTS y los incluye en enfoques integradores para dar forma a la Tecnología y la innovación, basándose a su vez en procesos de innovación proporcionados por aquellos.

4 La traducción es nuestra. 


\section{El movimiento STEM}

El movimiento STEM se inicia en la década de los 90, con la National Science Foundation como principal organismo impulsor. Tras unos años de escasa repercusión social y también educativa (Friedman, 2005), desde 2009 ha experimentado una expansión global fundamentada en las necesidades sociales expuestas por la National Governor's Association, quien definió la promoción de una identidad STEM entre la ciudadanía como un medio para mantener la competitividad económica de EE.UU. frente a las potencias emergentes. La identidad STEM del alumnado, si nos referimos al ámbito educativo, o de la ciudanía, desde una perspectiva social más amplia, responde a la necesidad de que todos ellos: (1) comprendan la repercusión social de las Ciencias, la Tecnología, la Ingeniería y las Matemáticas; (2) estén capacitados para entender los avances y/o aportes sociales impulsados por las disciplinas STEM; y (3) manifiesten interés por estas disciplinas. Ello permitiría generar un sentimiento de pertenencia a una sociedad en la que las disciplinas STEM ocupan un rol esencial, el cual debería ser ajeno a razas, género y culturas (Brickhouse et al., 2000; Carlone y Johnson, 2007; Polman y Miller, 2010).

La alfabetización STEM es un término acuñado por el Washington STEM Study Group (2011) y que aparece imbricado al concepto identidad STEM. Esta organización define alfabetización STEM como la capacidad de identificar y aplicar contenidos de las áreas de conocimiento STEM para comprender y resolver aquellas situaciones problemáticas que no pueden ser resueltas desde un solo enfoque disciplinario. El desarrollo de esta nueva alfabetización implicaría que cada una de las disciplinas STEM incluya una serie de contenidos conceptuales, procedimentales y actitudinales, de forma que si el dominio de cada una de estas disciplinas STEM es necesario, también lo es la capacidad de reconocer y apreciar las conexiones que existen entre ellas. Esta integración de áreas de conocimiento implica obtener un producto final mayor que la suma de sus partes disciplinarias individuales. En la figura 2 hemos representado STEM como la intersección de las disciplinas de las que se alimenta.

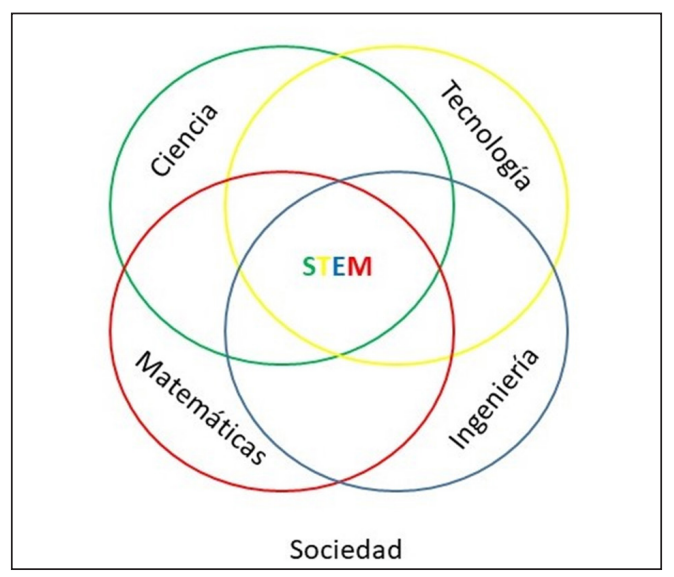

Figura 2. STEM como integración disciplinaria. Fuente: elaboración propia

La educación STEM resulta de la transposición al ámbito educativo del movimiento STEM, el cual emergió con claros propósitos políticos. Desde entonces las aproximaciones desde la investigación educativa a la educación STEM han sido múltiples, originando una amalgama de definiciones sobre este término tan diferentes entre sí que la han guiado hacia la ambigüedad. Tan es así que autores como:

- Kelley y Knowles (2016), Moore et al. (2014) y Sanders (2009) entienden la educación STEM como un enfoque de enseñanza que integra dos o más disciplinas STEM, 
utilizando contextos reales y con la intención de vincular estas disciplinas para mejorar el aprendizaje del alumnado.

- Shaughnessy (2013) ha definido a la educación STEM como la resolución de problemas basados en conceptos y procedimientos de Ciencias y Matemáticas, que incorporan las estrategias aplicadas en Ingeniería y el uso de Tecnología.

- Sanders y Wells (2006) puntualizan que se trata de una educación STEM integrada, tratándose de un enfoque de enseñanza basado en el diseño tecnológico o de Ingeniería, que integra intencionalmente contendidos conceptuales y procedimentales de la educación científica y/o matemática con los conceptos de naturaleza práctica propios de la educación tecnológica y de la Ingeniería.

- Martín-Páez, Aguilera, Perales-Palacios y Vílchez-González (2019) abogan por concebir la educación STEM como un enfoque de enseñanza en el que se integran, con mayor o menor grado, contenidos conceptuales y procedimentales propios de la Ciencia, la Tecnología, la Ingeniería y las Matemáticas.

A pesar de este amplio espectro de concepciones sobre la educación STEM, Bybee (2013) destaca que este enfoque de enseñanza contiene una evidente naturaleza interdisciplinar centrada en la resolución de problemas. En este sentido, en aras de contribuir a la clarificación didáctica y pedagógica de la educación STEM, MacKinnon, Rawn, Cressey y He (2017) proponen las siguientes definiciones para facilitar el diseño de un currículo STEM:

- La Ciencia como una "forma de conocimiento" que busca comprender el mundo que nos rodea.

- La Tecnología como una "forma de adaptación" que necesariamente considera los impactos sociales.

- Ingeniería como una "forma de diseñar/crear dispositivos" para responder a problemas reales.

- Las Matemáticas como una "forma de expresar una comprensión/análisis del mundo y los problemas auténticos a través de los números".

Al igual que el movimiento CTS, el movimiento STEM también ha experimentado evoluciones en su acrónimo. Así, autores como Land (2013) y Maeda (2013) manifiestan la oportunidad de integrar las artes (A) en la educación STEM a fin de permitir al alumnado: (1) aunar el pensamiento convergente (característico de las disciplinas STEM) y divergente (habitual en las disciplinas artísticas y humanísticas) en la resolución de problemas reales; (2) la creación de significado personal; y (3) la automotivación. De modo que, ante la "neblina didáctica" que han generado las múltiples concepciones sobre educación STEM -principalmente por la dificultad de integrar las cuatro disciplinas a la vez-, ha emergido un enfoque de enseñanza paralelo denominado educación STEAM; el cual ha heredado las limitaciones de su predecesor y dividido los esfuerzos de la comunidad educativa e investigadora por disipar las incertidumbres que rodean a estos enfoques de enseñanza.

Una vez contextualizado y definido el término educación STEM, podríamos sintetizar sus principales características en: (a) se presenta como un enfoque de enseñanza que integra Ciencia, Tecnología, Ingeniería y Matemáticas; (b) orientado a la resolución de problemas, preferentemente reales, a fin de desarrollar una alfabetización STEM; como consecuencia de lo anterior (c) persigue la promoción de una identidad STEM entre la ciudadanía que le permita comprender la repercusión social de las Ciencias, la Tecnología, la Ingeniería y las Matemáticas, así como participar de forma activa y crítica en la toma de decisiones; y (d) sus objetivos de aprendizaje interpelan al reconocimiento de los vínculos existentes entre las disciplinas STEM y su aplicación en la resolución de problemas reales, conocimientos y habilidades imprescindibles para cumplir lo descrito en los apartados (b) y (c). 
Dadas las características de la educación STEM, las metodologías de enseñanza activas parecen situarse como aquellas ideales para implementar este modelo de enseñanza. Así, el aprendizaje basado en proyectos (Domènech-Casal, 2018) o aquel basado en problemas (Lou, Shih, Diez, y Tseng, 2011) han sido utilizados para implementar propuestas didácticas de enfoque STEM (Martín-Páez et al., 2019).

\section{¿Cómo se relacionan CTS y STEM?}

Tras la somera explicitación de los fundamentos de los movimientos CTS y STEM, vamos a sintetizar las relaciones establecidas en una revisión de la literatura realizada a partir de estas dos palabras clave:

- Zoller (2013) defiende una traslación de los objetivos CTSA con el consenso de los responsables educativos y educadores STEM en forma de programas educativos, currículos, cursos, enseñanza, aprendizaje y estrategias de evaluación.

- Siu, Wong y Feng (2010) destacan que, en el pasado siglo, educadores e investigadores en Tecnología sugirieron que esta última debería ser enseñada de una forma integrada junto con otras materias (CTS y STEM).

- Para MacKinnon, Rawn, Cressey y He (2017), el movimiento educativo STEM ha evolucionado a partir de la iniciativa curricular integrada CTS de la década de 1990 y la noción de que la educación tecnológica debería ser más que una aventura de resolución de problemas, tras la tradición centrada en la formación vocacional emanada de la revolución industrial.

- Yalaki (2016) afirma que, aunque el movimiento CTSA perdió importancia en los últimos años, ha sido uno de los intentos de reforma educativa más importantes en la historia de la educación científica. Hoy día, conceptos como problemas socio-científicos o STEM son más frecuentes.

- Gordillo (2019) reivindica la vigencia del movimiento CTS frente a la "vaporosidad" de STEM o STEAM y, al contrastar ambas corrientes, critica que este último ignore el objetivo del primero consistente en "aprender a valorar y aprender a participar en las decisiones relacionadas con el desarrollo tecnocientífico", así como su excesiva atención al funcionamiento y desarrollo de los dispositivos tecnológicos, desatendiendo la comprensión del comportamiento de la naturaleza

A partir de los apartados previos, creemos estar en condiciones de visualizar una comparación entre ambos tópicos (CTS y STEM) a partir de las dimensiones que los caracterizan (Tabla 2).En cuanto al origen de ambos movimientos, se evidencia cómo el movimiento STEM emerge cuando aquel que le precedió cronológicamente -movimiento CTS- logró consolidarse como un enfoque útil y necesario para enseñar Ciencias. Esto podría hacernos pensar en la oportunidad del surgimiento del movimiento STEM (Domènech-Casal, 2019), el cual podría representar: (1) una "moda educativa" en aras de captar financiación y repercusión para instituciones educativas, proyectos y/o grupos de investigación; o (2) una evolución o revolución -incidiremos en ello más tarde- que parte del movimiento CTS a fin de adaptar la enseñanza y el aprendizaje a un mundo global y necesariamente interdisciplinar. Sin duda, a nuestro juicio, la primera acepción resulta de una mala interpretación del movimiento STEM que busca rentabilizar económicamente el potencial de una "moda educativa" $y$, además, oportunista dado que aprovecha la ambigüedad con la que se ha definido la educación STEM y la escasa formación del profesorado sobre este enfoque de enseñanza. Así, no es de extrañar encontrar kits de robótica con el eslogan "educación STEM" y, en consecuencia, experiencias educativas con la misma palabra clave que simplemente utilizan estos artefactos. 
Tabla 2. Comparación entre las características generales de los movimientos CTS y STEM

\begin{tabular}{|c|c|c|}
\hline Dimensiones & Movimiento CTS & Movimiento STEM \\
\hline $\begin{array}{l}\text { Origen } \\
\text { cronológico }\end{array}$ & $\begin{array}{l}\text { - Inicio: artículo de Gallagher (1971) } \\
\text { en la revista Science Education (de } \\
\text { acuerdo a Aikenhead, 2003). } \\
\text { - Consolidación: informe "Technolo- } \\
\text { gy for All Americans: A Rationale and } \\
\text { Structure for the Study of Technolo- } \\
\text { gy" (USA, 1996). }\end{array}$ & $\begin{array}{l}\text { - Inicio: National Science Foundation } \\
\text { (USA, 1998). } \\
\text { - Consolidación: creación de un gra- } \\
\text { do en educación STEM en la Univer- } \\
\text { sidad de Virginia Tech (Friedman, } \\
\text { 2005). }\end{array}$ \\
\hline Naturaleza & Humanística-Curricular & Política-Curricular \\
\hline Objetivos & $\begin{array}{l}\text { - Enseñanza: facultar a los ciudada- } \\
\text { nos para que comprendan las ideas } \\
\text { principales de la Ciencia y tomen } \\
\text { decisiones informadas sobre temas } \\
\text { sociales relacionados con la Ciencia y } \\
\text { la Tecnología. } \\
\text { - Aprendizaje: resolver problemas } \\
\text { sociales, desarrollar el pensamiento } \\
\text { crítico y valores de participación ciu- } \\
\text { dadana. }\end{array}$ & $\begin{array}{l}\text { - Enseñanza: facultar a los ciudada- } \\
\text { nos para que entiendan los avances } \\
\text { y/o aportes sociales impulsados por } \\
\text { las disciplinas STEM y para que mani- } \\
\text { fiesten interés por estas disciplinas. } \\
\text { - Aprendizaje: conseguir la alfabeti- } \\
\text { zación STEM. }\end{array}$ \\
\hline $\begin{array}{l}\text { Metodología } \\
\text { de enseñanza }\end{array}$ & $\begin{array}{l}\text { - Análisis de fuentes de información. } \\
\text { - Aprendizaje basado en proyectos. } \\
\text { - Estudios de caso. } \\
\text { - Actividades extracadémicas... }\end{array}$ & $\begin{array}{l}\text { - Aprendizaje basado en proyectos. } \\
\text { - Aprendizaje basado en problemas. }\end{array}$ \\
\hline
\end{tabular}

En el caso de los objetivos de la Tabla 2, también podríamos incluir la perspectiva competencial, tan en boga hoy día desde el surgimiento del Espacio Europeo de Educación Superior; en este caso CTS pondría mayor acento en la competencia ciudadana y STEM en la laboral. Ello visibilizaría el acento humanístico que atribuimos a la naturaleza CTS frente al político de STEM. A este respecto no pretendemos afirmar que CTS careciera de una perspectiva política, si se considera como tal la búsqueda institucional de una alfabetización científica de la ciudadanía para su participación activa en las decisiones que le competen, sino que aquella queda desdibujada frente al evidente impulso del poder político al movimiento STEM para ganar en competitividad económica.

La naturaleza de ambos movimientos es uno de los puntos diferenciadores principales, debido a que el movimiento CTS se origina como una necesidad de mejorar el currículo desde una perspectiva integradora, una oportunidad de contextualizar la enseñanza de las Ciencias en situaciones problemáticas sociales y de humanizar las Ciencias con aportes filosóficos (Aikenhead, 2003; Lawrence et al., 2001). Así la educación CTS se ha definido como un enfoque interdisciplinar que busca explorar y comprender, por un lado, las formas en que la Ciencia y la Tecnología inciden en la cultura, los valores y las instituciones modernas y, por otro, cómo los valores y la cultura influyen en la Ciencia y la Tecnología (Mansour, 2009). Por tanto, si tomamos la naturaleza curricular de la educación CTS como punto de partida y atendemos a la naturaleza filosófico-curricular de las SSI y político-curricular de la educación STEM, podríamos entrever estos dos últimos enfoques de enseñanza como dos evoluciones diferenciadas del primero (Figura 3): 
- La educación basada en SSI se presenta como un enfoque de enseñanza que profundiza el movimiento CTS, al centrar decididamente el proceso de enseñanza-aprendizaje en el desarrollo de valores morales, éticos y culturales que permitan al alumnado juzgar críticamente los avances tecno-científicos (Zeidler et al., 2005).

- La educación STEM adopta una línea evolutiva opuesta, orientada al desarrollo competencial del alumnado desde una perspectiva integradora y con claros fines políticos. En este sentido, Aikenhead (2003) abogó que para cambiar el status quo del currículo de Ciencias (también el de Tecnología, Ingeniería y Matemáticas) se requieren intervenciones políticas basadas en políticas creativas e irracionales, además de innovaciones curriculares basadas en fundamentos filosóficos racionales.

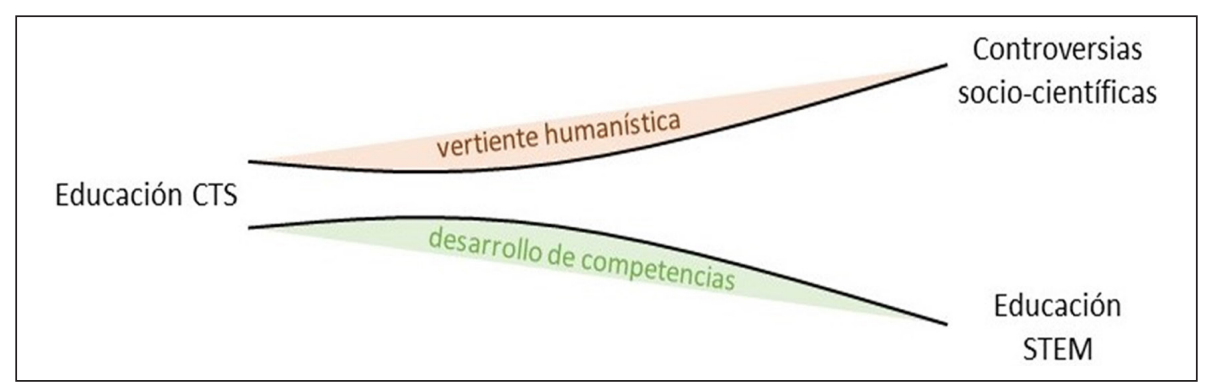

Figura 3. Evolución desde CTS hacia STEM y controversias socio-científicas. Fuente: elaboración propia

Vistos los matices evolutivos que presenta STEM respecto a CTS, en la Tabla 3 ahondamos en la naturaleza didáctica de ambos movimientos.

Tabla 3. Comparación entre las orientaciones didácticas de CTS y STEM

\begin{tabular}{|l|l|l|}
\hline Dirigidas a & $\begin{array}{l}\text { Orientaciones CTS (adaptadas de } \\
\text { Lawrence et al., 2001) }\end{array}$ & Orientaciones STEM \\
\hline \multirow{2}{*}{ Currículo } & $\begin{array}{l}\text { Centrado en cuestiones sociales y/o } \\
\text { culturales de índole tecno-científica. }\end{array}$ & $\begin{array}{l}\text { Centrado en generar una identidad } \\
\text { STEM en el alumnado. }\end{array}$ \\
\hline \multirow{7}{*}{ Proceso de E-A } & $\begin{array}{l}\text { Enfatiza la adaptación humana y fu- } \\
\text { turos alternativos. }\end{array}$ & $\begin{array}{l}\text { Destaca la repercusión social de las } \\
\text { disciplinas STEM. }\end{array}$ \\
\cline { 2 - 4 } & $\begin{array}{l}\text { La información está en el contexto } \\
\text { del estudiante como persona en un } \\
\text { entorno cultural/social. }\end{array}$ & $\begin{array}{l}\text { La información está en los contenidos } \\
\text { (conceptuales, procedimentales y ac- } \\
\text { titudinales) de las disciplinas STEM. }\end{array}$ \\
\cline { 2 - 4 } & $\begin{array}{l}\text { Atiende a las dimensiones de valor, } \\
\text { ética y moral de los problemas. }\end{array}$ & $\begin{array}{l}\text { Atiende a la capacidad de transferir } \\
\text { conocimiento a contextos diferentes. }\end{array}$ \\
\hline \multirow{2}{*}{$\begin{array}{l}\text { Rol del } \\
\text { alumnado }\end{array}$} & $\begin{array}{l}\text { Busca y analiza información. } \\
\text { Ve la Ciencia como una forma de } \\
\text { afrontar los problemas de la vida co- } \\
\text { tidiana. }\end{array}$ & $\begin{array}{l}\text { Busca y analiza información para } \\
\text { crear contenido propio. }\end{array}$ \\
\cline { 2 - 4 } & $\begin{array}{l}\text { Identifica problemas acerca de ellos } \\
\text { mismos o de su comunidad y asume } \\
\text { la responsabilidad de resolverlos uti- } \\
\text { lizando la Ciencia. }\end{array}$ & $\begin{array}{l}\text { Deconstruye la situación problemáti- } \\
\text { ca, identificando qué contenidos de } \\
\text { las disciplinas STEM son necesarios } \\
\text { para aplicarlos en una posible solu- } \\
\text { ción. }\end{array}$ \\
\hline
\end{tabular}


Tabla 3. Comparación entre las orientaciones didácticas de CTS y STEM. Continuación

\begin{tabular}{|l|l|l|}
\hline Dirigidas a & $\begin{array}{l}\text { Orientaciones CTS (adaptadas de } \\
\text { Lawrence et al., 2001) }\end{array}$ & Orientaciones STEM \\
\hline $\begin{array}{l}\text { Rol del } \\
\text { profesorado }\end{array}$ & $\begin{array}{l}\text { Considera sus propias creencias y va- } \\
\text { lores relacionados con los problemas } \\
\text { sociales y el impacto que estos tienen } \\
\text { en la enseñanza. }\end{array}$ & $\begin{array}{l}\text { Selecciona o diseña situaciones pro- } \\
\text { blemáticas reales y considera los } \\
\text { contenidos (conceptuales, procedi- } \\
\text { mentales y actitudinales) de las disci- } \\
\text { plinas STEM útiles para su resolución. }\end{array}$ \\
\cline { 2 - 3 } & $\begin{array}{l}\text { Desarrolla un marco flexible para el } \\
\text { aprendizaje y establece preguntas } \\
\text { amplias para guiar el aprendizaje. }\end{array}$ & $\begin{array}{l}\text { Establece preguntas con el objetivo } \\
\text { de guiar el aprendizaje y explicitar los } \\
\text { vínculos entre las disciplinas STEM. }\end{array}$ \\
\cline { 2 - 3 } & $\begin{array}{l}\text { Otorga al alumnado un rol protago- } \\
\text { nista durante el proceso de E-A. }\end{array}$ & $\begin{array}{l}\text { Centra el proceso de E-A en el alum- } \\
\text { nado. }\end{array}$ \\
\hline
\end{tabular}

\section{Consideraciones finales}

Tras lo expresado hasta aquí y, a la vista de las Tablas 2 y 3, creemos estar en condiciones de dar respuesta al objetivo que nos planteamos en este trabajo, es decir, fundamentar la comparación entre los movimientos CTS y STEM que, en cierta medida, han acaparado la atención de educadores e investigadores en Didáctica de las Ciencias durante las últimas décadas a nivel internacional.

Ante la pregunta de si ese contraste puede desembocar en una evolución, revolución o disyunción, estimamos que estamos frente a una evolución pero con escasas aportaciones originales, pudiendo resumirlas en:

- El origen STEM posee unas connotaciones políticas y económicas más marcadas que las de CTS.

- En consonancia con ese origen, STEM trata de incrementar las vocaciones y las competencias científico-tecnológicas y matemáticas que ayuden a mejorar la competitividad de los países.

- Frente a CTS, que enfatiza los vínculos de la Ciencia y la Tecnología con la Sociedad, STEM lo hace sobre las relaciones entre las disciplinas integradas en el acrónimo.

- El papel de la Tecnología en CTS es más subsidiario que en el caso de STEM, donde adquiere un protagonismo mayor.

Esta evolución desde CTS a STEM también repercute en la transposición de estos movimientos al aula, de forma que presentan las siguientes diferencias:

- El currículo basado en la educación STEM se centra en generar una identidad STEM en el alumnado, frente aquel de orientación CTS que atendía a las cuestiones sociales de índole científico-tecnológica, junto con el aprendizaje de la Ciencia (en menor medida el de la Tecnología).

- El proceso de enseñanza-aprendizaje (E-A), en el caso de la educación CTS, guiaba al alumnado hacia la adquisición de valores a fin de juzgar problemas sociales en los que la Ciencia y la Tecnología estaban presentes. En cambio, la educación STEM genera un proceso de E-A dirigido a la transferencia del conocimiento científico, tecnológico, ingeniero y matemático.

A la vista de estas consideraciones, solo cabe preguntarse si estas humildes aportaciones de STEM frente a CTS son suficientes como para concederle al primero ese papel tan relevante que parece ir adquiriendo entre las instituciones y la comunidad científica y docente 
actual. Al hilo de esta apreciación, Aikenhead (2003) ya atisbó que, teniendo en cuenta la historia del movimiento CTS, aparecerían otras consignas o eslóganes propiciados por los innovadores educativos, bien por ajustes a los cambios culturales y sociales, bien por distanciarse de eslóganes pasados o por la necesidad de ser vistos haciendo algo diferente. Esto le llevó a expresarlo como "Educación Ciencia-Tecnología-Sociedad (CTS): una buena idea como quiera que se le llame" (Aikenhead, 2003).

En cualquier caso queda pendiente el desarrollo de un marco teórico que sustente a la educación STEM desde la psicodidáctica a fin de aunar criterios en lo que a la transposición al aula de este enfoque se refiere, además de reformas de gran calado como es la formación de formadores STEM (tal cual ocurrió en su momento con CTS), aunque se hayan dado unos tímidos pasos. Mientras no se habiliten títulos universitarios (o de posgrado) que articulen un conocimiento disciplinar y didáctico con una cierta entidad, se generen redes nacionales de profesorado y los currículos escolares no sean lo suficientemente flexibles como para admitir estos enfoques sin grandes traumas, es de prever (al menos en nuestro país) un futuro poco halagüeño, más allá de incorporar algunas experiencias puntuales y con vinculaciones interdisciplinares limitadas. Por el contrario, si la recuperación de CTS o STEM para la agenda educativa presente contribuye a mover los cimientos de una enseñanza de la Ciencia anclada en una visión de la misma y unos métodos de enseñanza arcaicos, bienvenido sea el debate.

\section{Agradecimientos}

A la Universidad de Granada por la financiación de la beca-contrato puente concedida a David Aguilera para completar su formación posdoctoral. También al Ministerio de Ciencia, Innovación y Universidades por la financiación del proyecto PGC2018-095765B-I00 (PROFESTEM).

Los autores desean expresar su reconocimiento a los Profs. José Antonio Acevedo Díaz y Antonio García Carmona por sus valiosos comentarios.

\section{Referencias bibliográficas}

Acevedo, J.A. (1995). Educación tecnológica desde una perspectiva CTS. Una breve revisión del tema. Alambique, 3, 75-84.

Acevedo, J.A. (1996). La Tecnología en las relaciones CTS. Una aproximación al tema. Enseñanza de las Ciencias, 14(1), 35-44.

Acevedo, J. A. (1997). Ciencia, Tecnología y Sociedad (CTS). Un enfoque innovador para la enseñanza de las Ciencias. Revista de Educación de la Universidad de Granada, 10, 269-275.

Aikenhead, G.S. (2003). STS education: A rose by any other name. En R. Cross (Ed.) A vision for science education: Responding to the work of Peter J. Fensham (pp. 59-75). Saskatoon, Canadá: Routledge Press 5 .

Bernal, J.D. (1967). Historia Social de la Ciencia. Barcelona: Ed. Península.

Brickhouse, N.W., Lowery, P., y Schultz, K. (2000). What kind of a girl does science? The construction of school science identities. Journal of Research in Science Teaching, 37(5), 441-458

5 Existe una versión en español: Educación Ciencia-Tecnología-Sociedad (CTS): una buena idea como quiera que se le llame. Educación Química, 16(2), 114-124 (2005). 
Bybee, R.W. (2013). The case for STEM education challenges and opportunities. National STEM Teachers Association.

Carlone, H.B. y Johnson, A. (2007). Understanding the Science Experiences of Successful Women of Color: Science Identity as an Analytic Lens. Journal of Research in Science Teaching, 44(8), 1087-1218. DOI: https://doi.org/10.1002/tea.20237

Chowdhury, M.A. (2016). The Integration of Science-Technology-Society/ScienceTechnology-Society-Environment and Socio-Scientific-Issues for Effective Science Education and Science Teaching. Electronic Journal of Science Education, 20(5), 19-38.

Domènech-Casal, J. (2018). Aprendizaje Basado en Proyectos en el marco STEM: componentes didácticas para la Competencia Científica. Ápice. Revista de Educación Cientifica, 21(2), 29-42. DOI: https://doi.org/10.17979/arec.2018.2.2.4524

Domènech-Casal, J. (2019). STEM: Oportunidades y retos desde la Enseñanza de las Ciencias. Universitas Tarraconensis. Revista de Ciències de l'Educació, 1(2), 154-168. DOI: https://doi.org/10.17345/ute.2019.2.2646

Fensham, P.J. (2016). The Future Curriculum for School Science: What Can Be Learnt from the Past? Research in Science Education, 46(2), 165-185. DOI: https://doi. org/10.1007/s11165-015-9511-9

Friedman, T.L. (2005). The World is flat. A brief history of the twenty-first century. Farrar, Straus and Giroux.

Gallagher, J.J. (1971). A broader base for science education. Science Education, 55, 329338.

García-Carmona, A. (2008). Relaciones CTS en la educación científica básica. I. Un análisis desde los textos escolares en la enseñanza de la electrónica. Enseñanza de las Ciencias, 26(3), 375-388.

García-Carmona, A. y Criado, A.M. (2008). Enfoque CTS en la enseñanza de la Energía Nuclear: análisis de su tratamiento. Enseñanza de las Ciencias, 26(1), 107-124.

Gordillo, M. Educación con enfoque CTS. Cátedra CTS+l. Perú. Recuperado de: https:// docplayer.es/57974752-Catedra-cts-i-peru-modulo-7-profesor-mariano-martingordillo.html

Gordillo, M. (2019). STEAM(E). Escuela. Recuperado de: https://maculammg.blogspot. com/2019/10/steame.html

Grunwald, A. (2011). Responsible Innovation: Bringing together Technology Assessment, Applied Ethics, and STS research. Enterprise and Work Innovation Studies, 7, 9 - 31.

Kelley, T.R. y Knowles, J.G. (2016). A conceptual framework for integrated STEM education. International Journal of STEM Education, 3(11), 1-11.

Kolst $\varnothing$, S.D. (2001). 'To trust or not to trust...'- pupils' ways of judging information encountered in a socio-scientific issue. International Journal of Science Education, 23(9), 877- 901. DOI: https://doi.org/10.1080/09500690010016102

Kuhn, T. (1961). La estructura de las revoluciones científicas. México: Fondo de Cultura Económica.

Land, M.H. (2013). Full STEAM ahead: The benefits of integrating the arts into STEM. Procedia Computer Science, 20, 547-552. DOI: https://doi.org/10.1016/j. procs.2013.09.317 
Lawrence, C., Yager, R., Sowell, S., Hancock, E., Yalaki, Y. y Jablon, P. (2001). The Philosophy, Theory and Practice of Science-Technology-Society Orientations. Annual Meeting of the Association for the Education of Teachers in Science, Costa Mesa, CA. Recuperado de: https://files.eric.ed.gov/fulltext/ED472905.pdf

Lou, S.J., Shih, R.C., Diez, C.R. y Tseng, K.H. (2011). The impact of problem-based learning strategies on STEM knowledge integration and attitudes: an exploratory study among female Taiwanese senior high school students. International Journal of Technology and Design Education, 21(2), 195-215. DOI: https://doi.org/10.1007/ s10798-010-9114-8

MacKinnon, G.R., Kate, G., Rawn, E., Cressey, J. y He, W. (2017). Employing STEM Curriculum in an ESL Classroom: A Chinese Case Study. K-12 STEM Education, 3(1), 143-155. DOI: http://dx.doi.org/10.14456/k12stemed.2017.1

Maeda, J. (2013). STEM + Art = STEAM. The STEAM Journal, 1(1), Article 34. DOI: http:// dx.doi.org/10.5642/steam.201301.34

Mansour, N. (2009). Science-technology-society (STS). A new paradigm in science education. Bulletin of Science, Technology \& Society, 29(4), 287-297. DOI: http:// dx.doi.org/10.1177/0270467609336307

Martín-Páez, T., Aguilera, D., Perales-Palacios, F.J. y Vílchez-González, J.M. (2019). What are we talking about when we talk about STEM education? A systematic review of literature. Science Education, 103, 799-822. DOI: http://doi.org/10.1002/sce.21522

Montovani, F. (2020). Noosfera. Recuperado de: http://teilhard.net/noosfera

Moore, T., Stohlmann, M., Wang, H., Tank, K., Glancy, A. y Roehrig, G. (2014). Implementation and integration of engineering in K-12 STEM education. En S. Purzer, J. Strobel, y M. Cardella (Eds.), Engineering in Pre-College Settings: Synthesizing Research, Policity, and Practices (pp. 35-60). Indiana, USA: Purdue University Press.

Morin, E. (1990). Introducción al pensamiento complejo. Barcelona: Gedisa.

Polman, J.L. y Miller, D. (2010). Changing Stories: Trajectories of Identification among African American Youth in a Science Outreach Apprenticeship. American Educational Research Journal, 47(4), 879-918. DOI: https://doi.org/10.3102/0002831210367513

RRI Tools (2020). Recuperado de: https://www.rri-tools.eu/es/about-rri

Sanders, M. (2009). STEM, STEM education, STEM mania. Technology Teacher, 68(4), 20-26.

Sanders, M. y Wells, J. (2006). Integrative STEM Education Course Syllabi \& Instructional Materials: STEM Education Foundations. En STEM Education Trends \& Issues, STEM Education Seminar.

Shaughnessy, J.M. (2013). Mathematics in a STEM Context. Mathematics Teaching in the Middle School, 18(6), 324.

Siu, K.W.M., Wong, Y.L. y Feng, W.W. (2010). Why fail? Experience of technology education in Hong Kong. World Transactions on Engineering and Technology Education, 8(2), 231-236.

Solbes, J. y Vilches, A. (1989). Interacciones Ciencia/Técnica/Sociedad: un instrumento de cambio actitudinal. Enseñanza de las Ciencias, 7(1), 14-20.

Strieder, R.B., Bravo-Torrija, B. y Gil-Quílez, M.J. (2017) Ciencia-Tecnología-sociedad: ¿Qué estamos haciendo en el ámbito de la investigación en educación en Ciencias? 
Enseñanza de las Ciencias, 35(3), 29-49. DOI: https://doi.org/10.5565/rev/ ensCiencias.2232

Toulmin, S. (1972). La comprensión humana. Madrid: E. Alianza.

Vilches, A., Gil D. y Praia, J. (2011). De CTS a CTSA: Educação por um futuro sustentável. En W. Santos y D. Auler (Eds.), CTS e Educação científica, desafio, tendências e resultados de pesquisa, pp.161-184. Brasilia: Editora UnB.

Vilches, A. y Gil, D. (2003): Construyamos un futuro sostenible. Diálogos de supervivencia. Madrid: Cambridge University Press.

Yalaki, Y. (2016). Improving University Students' Science-Technology-Society-Environment Competencies. International Journal of Progressive Education, 12(1), 90-98.

Zeidler, D.L., Sadler, T.D., Simmons, M.L. y Howes, E.V. (2005). Beyond STS: A researchbased frame work for socio scientific issues education. Science Education, 89(3), 357-377. DOI: https://doi.org/10.1002/sce.20048

Zoller, U. (2011). Science and Technology Education in the STES Context in Primary Schools: What Should It Take? Journal of Science Education and Technology, 20(5), 444-453.

Zoller, U. (2012). Science Education for Global Sustainability: What is necessary for Teaching, Learning and Assessment Strategies? Journal of Chemical Education, 89, 297-300. DOI: https://doi.org/10.1021/ed300047v

Zoller, U. (2013). Science, Technology, Environment, Society (STES) Literacy for Sustainability: What Should it Take in Chem/Science Education? Educación Química, 24(2), 207-21. DOI: https://doi.org/10.1016/S0187-893X(13)72464-9

Zoller, U. y Nahum, T.L. (2012). From Teaching to 'Know'-to Learning to 'ink' in Science Education. En Fraser, B., Tobin, K. y McRobbie, C. (Eds.), 2nd International Handbook of Science Education, 2a Ed., 1(16), 209-330. Nueva York: Springer International Handbook of Education. 
